\title{
A OFTALMOLOGIA PREVENTIVA NA ABORDAGEM DOS FAMILIARES DE PORTADORES DE GLAUCOMA: RELATO DE EXPERIÊNCIA
}

\author{
Aganeide Castilho - Palitot \\ Beatriz Wanderley Gayoso de Lima" \\ Daniel Virgulino Leite ${ }^{\text {II }}$ \\ Fábio Carvalho Tavares Emídio " \\ Otacílio Francisco Paraguay Figueiredo " \\ Juliana de Oliveira Castelo Branco II
}

\begin{abstract}
RESUMO
Campanhas de prevenção da cegueira, ou de promoção de saúde ocular, têm sido realizadas por muitos grupos de saúde de instituições de ensino superior pois, ao mesmo tempo que asseguram a melhoria da qualidade de vida da população, através da conscientização e do maior conhecimento sobre as doenças oftalmológicas, também proporcionam a integração entre alunos e médicos, junto à comunidade carente. Dessa forma, o objetivo deste trabalho é a explanação da prática da prevenção em saúde, identificando a importância do papel do doente glaucomatoso e de sua família como grandes disseminadores da prevenção do glaucoma. Este é um estudo descritivo, do tipo relato de experiência acerca das atividades do projeto de extensão intitulado "Glaucoma em Família". Este relato foi construído durante os dois semestres letivos do ano de 2017, quando os seus integrantes puderam exercer atividades de conscientização sobre a avaliação ocular, visto que o glaucoma é uma doença de caráter genético e de melhor manejo quando detectado precocemente. Os extensionistas utilizaram a estratégia da abordagem por vídeos e imagens ilustrativas sobre o glaucoma e seus fatores de risco, esclarecendo e tirando dúvidas dos doentes e seus familiares, que estavam no ambulatório de oftalmologia, acerca dos métodos necessários para sua prevenção e controle. As ações desenvolvidas contribuíram para uma melhor conscientização da população, pois foi vista uma boa interação e atenção do público-alvo. No entanto, também revelou a real existência de grande desinformação sobre a doença glaucomatosa, suas complicações, tratamento e principalmente prevenção. Portanto, esse trabalho concluiu que ainda são necessárias mais medidas e campanhas de prevenção primária e secundária visando a divulgação de conhecimentos, relativos ao glaucoma, aos próprios doentes e suas famílias, como forma de prevenção da perda visual.
\end{abstract}

\section{PALAVRAS-CHAVE}

Glaucoma. Prevenção primária. Promoção de saúde.

\section{INTRODUÇÃO}

A educação, a promoção e a medicina preventiva em saúde é um conjunto elaborado e coerente de intervenções sobre o indivíduo, ou grupo comunitário que deve ajudá-lo a querer, poder e saber escolher e adotar de forma responsável, livre e esclarecida as atitudes e os comportamentos adequados para favorecer a sua saúde ${ }^{1}$.
Existem dados suficientes que sugerem que programas de cuidados de saúde, realizados junto de pacientes com doenças crônicas, a exemplo do glaucoma, melhoram o seu estado de saúde e reduzem os custos. O aumento da conscientização e do conhecimento do doente glaucomatoso, da sua família e da sociedade em geral, acerca do 
glaucoma, leva a um aumento da compreensão e aceitação da importância do tratamento de tal condição com a consequente redução dos casos de cegueira ${ }^{1}$.

O glaucoma é uma doença crônica caracterizada por uma progressiva alteração do nervo óptico, conduzindo à perda visual. É a segunda principal causa de cegueira no mundo, ficando atrás somente da catarata. Esses danos visuais progridem gradualmente e, frequentemente, passam despercebidos ao paciente. Assim, a prevenção, através da detecção precoce e o controle da doença, são primordiais para prevenir a cegueira irreversível do glaucoma. Além disso, os custos com os tratamentos na saúde pública têm sido uma constante preocupação dos gestores públicos e, na oftalmologia, o glaucoma em um nível avançado de comprometimento no paciente, tem um impacto considerável para o Sistema Único de Saúde ${ }^{1}$.

Por ser uma doença com grande fator hereditário, uma correta disseminação da medicina preventiva, perante os familiares do paciente glaucomatoso, tem uma importância ainda maior nesse elo entre a família e o doente no combate

\section{METODOLOGIA}

Este estudo é formatado como um relato de experiência descritivo, referente às ações de educação em saúde, desenvolvidas por extensionistas do Projeto Glaucoma em Família da Faculdade de Medicina e Enfermagem Nova Esperança (FAMENE).

Os discentes foram subdivididos em grupos, os quais cumpriram a demanda da carga horária de 4 horas semanais referentes às quintas-feiras ou sextas-feiras. As atividades de promoção de saúde foram realizadas nos ambulatórios do centro médico da Faculdade de Medicina e Enfermagem Nova Esperança (FAMENE), nas a neuropatia óptica glaucomatosa ${ }^{2}$. O esclarecimento dos familiares, através de projetos e ações por grupos de saúde, tem se revelado uma importante ferramenta para diminuir os altos índices de perda visual em parentes de pacientes glaucomatosos que não têm a devida orientação sobre essa doença ${ }^{2}$. Essas campanhas de prevenção da cegueira e de promoção da saúde ocular devem ser fomentadas e realizadas por alunos de todas as instituições de saúde, porque ajuda a proporcionar a integração de alunos, médicos e residentes com a população mais carente que, na grande maioria dos casos, não tem atendimento preventivo, buscando ajuda apenas quando a cegueira está parcial ou totalmente instalada ${ }^{2}$.

O objetivo deste trabalho é a explanação da prática preventiva em saúde, através projeto de extensão científica intitulado "Glaucoma em Família", desenvolvido pelos estudantes de medicina, residentes de oftalmologia e os médicos preceptores, acerca das informações necessárias para garantir uma boa educação em prevenção à saúde em relação ao glaucoma.

unidades de João Pessoa(PB) e de Bayeux(PB). No dia 20 de outubro de 2017, os extensionistas também participaram do evento da Mega-Ação, no qual puderam adquirir conhecimentos na área da oftalmologia, acompanhar o atendimento ambulatorial de pacientes e promover uma conscientização efetiva da população sobre o glaucoma, fortalecendo o esclarecimento dos familiares e acompanhantes dos pacientes sobre a importância do exame oftalmológico periódico para o diagnóstico precoce da doença glaucomatosa e, portanto, contribuindo para a prevenção da cegueira. 
Para a execução das atividades, previamente, foi realizado um estudo embasado na literatura encontrada em fontes como Refração Aderbal de Albuquerque Alves (2014), Coleção CBO (Conselho Brasileiro de Oftalmologia), Kanski Oftalmologia Clínica Ed. Elsevier (2016), Manual de doenças oculares do Wills Eye Hospital Enlers (2015), bem como nas bases de dados dos sistemas Scielo e BVS (biblioteca virtual de saúde).

Os alunos foram orientados pela docente Aganeide Castilho-Palitot. As atividades tiveram como objetivo a promoção da educação em saúde da população acerca dos riscos e das principais características do glaucoma. Os discentes enfatizaram a importância do conhecimento sobre essa doença, pois, por ser de caráter incurável, a prevenção primária representa uma das principais estratégias para o controle da

\section{RESULTADOS E DISCUSSÕES}

A Promoção da Saúde, como conjunto de estratégias e formas de produzir saúde, no âmbito individual e coletivo, visando atender às necessidades sociais de saúde e garantir a melhoria da qualidade de vida da população, vincula-se à concepção expressa na Carta de Ottawa, documento em que 35 países ratificaram como principais ações aquelas que objetivem a redução das iniquidades em saúde, garantindo oportunidade para que todos os cidadãos sejam protagonistas no processo de produção da saúde e melhoria da qualidade de vida3.

A educação em saúde representa uma estratégia promissora para enfrentar os múltiplos problemas que afetam as populações humanas, partindo de uma concepção ampla do processo saúdedoença e de seus determinantes 4 . Assim, a prática da educação em saúde preconiza desenvolver nas pessoas juízo crítico e capacidade de intervenção sobre suas incidência da cegueira por glaucoma.

As ações foram direcionadas à população e aos seus familiares, caracterizadas pelo caráter humanizado e amplo da abordagem. A conduta foi adequada ao público-alvo, formatada por uma linguagem popular e acessível, fazendo o uso de explicações objetivas e com alguns aspectos lúdicos para a obtenção da atenção e visando otimizar a educação em saúde.

A campanha de conscientização realizada pelos extensionistas foi diversificada e foram feitas ações de panfletagens, produção de cartazes, palestras, explanações diretas e direcionamentos. Para a execução dessas ações, foram necessários materiais como panfletos, cartazes e cartilhas. Todos os materiais e o custo referente ao deslocamento dos extensionistas foram fornecidos pelo PROICE (FAMENE).

vidas e sobre o ambiente com o qual interagem 5. As informações e orientações devem ser expressas de maneira clara e sem restrições, de forma a garantir boa compreensão pelos sujeitos para que os objetivos e metas sejam alcançados 6 .

Através da promoção de práticas que proporcionam uma educação em saúde, os extensionistas puderam promover ações com o intuito de elucidar a população sobre o glaucoma. As exposições enfatizaram a importância da prevenção primária, principalmente pelo caráter incurável da doença. As atividades foram diversificadas, compostas por panfletagem, apresentações, palestras e explanações caracterizadas pela interação humanizada dos discentes com os pacientes. A linguagem utilizada foi apropriada para o público-alvo, otimizando um câmbio de conhecimento e, consequentemente, promovendo um aprendizado adequado. O glaucoma resulta principalmente do aumento da pressão intraocular que 
lesa o nervo óptico e conduz a perda progressiva da visão. É uma das principais causas de cegueira no mundo e, apesar de não ter cura, a cegueira pode ser evitada com diagnóstico precoce, através de exames oftalmológicos periódicos e controle da pressão intraocular. Outro aspecto relevante do glaucoma é que, mesmo nos países mais desenvolvidos, apenas 50\% dos doentes são efetivamente diagnosticados, considerando-se que em países pobres, ou em vias de desenvolvimento, esta percentagem seja muito inferior 7 .

Por ser uma doença silenciosa e de difícil diagnóstico precoce, o glaucoma é um problema grave de saúde pública. Um estudo retrospectivo e descritivo de 822 prontuários de pacientes atendidos pela primeira vez no Setor de Glaucoma da Universidade Federal de São Paulo - UNIFESP, no período de janeiro de 2000 a janeiro de 2001, indica que a maioria tratava-se de glaucomas avançados, sendo que $41,9 \%$ dos pacientes já apresentavam cegueira em pelo menos um olho ${ }^{8}$. A doença pode se desenvolver, durante meses ou anos, sem apresentar nenhuma característica clínica. Os sintomas só aparecem na fase mais avançada, quando o indivíduo começa a perder irreversivelmente a visão, principalmente o campo visual periférico 8 .

A estimativa atual é de que a patologia atingirá cerca de 70 milhões de pessoas em todo o mundo, isto é, 2 a 3\% da população mundial e que em 2020 esse número suba para 80 milhões ${ }^{8}$. Segundo o Ministério da Saúde, pessoas que têm parentes portado-

\section{CONSIDERAÇÕES FINAIS}

A atividade desenvolvida, durante o projeto de extensão, foi positiva uma vez que a população alvo interagiu e absorveu a linguagem, apresentada nas atividades do projeto, e se mostrou capaz de replicar as orientações recebidas para familiares e amigos, estimulando a procura do exame res de glaucoma, indivíduos com mais de 40 anos, pacientes com alto grau de miopia, diabéticos e hipertensos devem estar ainda mais atentos à realização dos testes de rotina. O diagnóstico é feito através do exame oftalmológico cuidadoso, com aferição da pressão intraocular, exame de fundo de olho e campo visual ${ }^{8}$.

Durante as ações, observou-se um predomínio de indivíduos que não tinham conhecimento sobre o glaucoma e seu caráter hereditário. A existência de história familiar de glaucoma sugere um componente genético dessa doença 9 . Portanto, é de suma importância a existência de iniciativas que promovam o conhecimento do indivíduo e de todo o seu núcleo familiar, caracterizando um projeto abrangente com ênfase na prevenção primária de toda a família. Os extensionistas puderam esclarecer vários aspectos da doença para a população, entre eles, enumeraram os principais fatores de risco, salientando a importância do paciente de continuar a transmissão de conhecimento para seus familiares devido ao potencial hereditário da doença.

Por se tratar de uma doença crônica e sem cura, o glaucoma pode ser controlado com o uso de medicamentos apropriados que normalizam a pressão intraocular e impedem que a doença avance provocando a perda irreversível da visão. Portanto, destaca-se a importância de campanhas e projetos educacionais com o intuito de elucidar a população acerca dessa doença e, consequentemente, promover a saúde.

oftalmológico para o diagnóstico precoce do Glaucoma. Também foi de grande valia para os alunos extensionistas, estimulando ainda mais a vontade de intensificar os estudos diante da percepção da necessidade da prevenção em saúde na área oftalmológica. 
Conclui-se especialmente que, diante da grande desinformação da população, principalmente dos familiares de pacientes portadores de glaucoma, há uma grande necessidade da manutenção da divulgação de informações sobre a prevenção e tratamento dessa doença nos consultórios e na comunidade, a fim de que se promova uma eficiente prevenção da perda visual na população em geral.

\title{
THE PREVENTIVE OPHTHALMOLOGY IN THE APPROACH OF GLAUCOMA CARRIERS FAMILY: EXPERIENCE REPORT
}

\begin{abstract}
Prevention campaigns for blindness or eye health promotion have been carried out by many health groups of higher education institutions, because by ensuring the improvement of the quality of life of the population through awareness and greater knowledge about ophthalmological diseases, it also provide the integration between students and doctors with the needy community. Thus, the objective of this work is to explain the practice of preventive medicine, identifying the importance of the role of the patiet with glaucoma and his family as major disseminators of glaucoma disease prevention. This is a descriptive study, a type of experience report about the activities of the extension project titled "Family Glaucoma" during the two academic semesters of the year 2017, in which its members were able to exercise awareness activities about the ocular evaluation, since glaucoma is genetic disease and it's better managed when is detected early. Extensionists used the strategy of approaching videos and illustrative images of glaucoma and its risk factors, clarifying the patients and their relatives who were in the ophthalmology outpatient clinic about the methods necessary for their prevention and control. The actions developed contributed to a better awareness of the population, as it was seen a good interaction and attention of the target public, but also revealed the real existence of great misinformation about glaucoma disease, its complications, treatment and mainly prevention. Therefore, this study concluded that further measures and campaigns of primary and secondary prevention are still very necessary in order to disseminate knowledge about glaucoma to patients and their families as a way to prevent visual loss.
\end{abstract}

\section{KEYWORDS}

Glaucoma. Prevention. Diseases

\section{REFERÊNCIAS}

1 Afonso ACPS. Glaucoma e educação para a saúde : influência de um programa de educação para a saúde junto de utentes glaucomatosos. [tese de mestrado em medicina]. Lisboa: Universidade de Lisboa; 2015. 295p.

2 Resnikoff S. et al. Global data on visual impairment in the year 2002. Bull World Health Organ, v. 82, n 11, p. 844-51, Nov. 2004.

3 Malta Deborah Carvalho, Morais Neto Otaliba Libanio, Silva Marta Maria Alves da, Rocha Dais, Castro Adriana Miranda de, Reis Ademar Arthur Chioro dos et al. Política Nacional de Promoção da Saúde (PNPS): capítulos de uma caminhada ainda em construção. Ciênc. saúde coletiva [Internet]. 2016 Junho. [Acesso:21Dez.2017]; 21(6): 1683-1694. Disponível em: http:// www.scielo.br/scielo.php?script=sci_arttext\&pid=S141381232016000601683\&lng=en.

4 Buss Paulo Marchiori. Promoção da saúde e qualidade de vida. Ciênc. saúde coletiva [Internet]. 2000. Acesso em: [21 Dez. 2017], 5 (1):163-177.Disponível em:http:// 
www.scielo.br/scielo.php?script=sci_arttext\&pid=S141381232000000100014\&lng=en

5 Levy, Sylvain Nahum et al. Educação em saúde: histórico, conceitos e propostas. In: Educação em saúde: histórico, conceitos e propostas. Brasil. Ministério da Saúde, 1997.

6 L'ABBATE, S. Educação em saúde: uma nova abordagem. Cad. Saúde Pública, Rio de Janeiro, v. 10, n. 4, Dec. 1994.

7 Carvalho, Clecilene Gomes et al. Glaucoma, inimigo oculto e perigoso da visão: Avaliação do nível de conhecimento dos diabéticos e hipertensos, do município de São Joaquim de Bicas (MG), sobre o glaucoma. e-Scientia [periódico da internet] Belo Horizonte 2010,
Acesso em: [21 Dez. 2017],3 (2):[p.02-12.].Disponível em: http://revistas.unibh.br/index.php/ dcbas/article/view/165/95

8 OLIVEIRA, Aldery de; PARANHOS JUNIOR, Augusto and PRATA JUNIOR, João Antônio. Características dos pacientes atendidos pela primeira vez no Setor de Glaucoma da Universidade Federal de São Paulo - UNIFESP. Arq. Bras. Oftalmol. [online]. 2003, vol.66, n.6, pp.785-790. ISSN 0004-2749.http://dx.doi.org/10.1590/ So004-27492003000700010.

9 Tavares, Ivan Maynart; MELLO, Paulo Augusto de Arruda. Glaucoma de pressão normal. Arquivos Brasileiros de Oftalmologia, 2005. 